\title{
IDRG-PTS scheme with low complexity for peak-to-average power ratio reduction in OFDM systems.
}

\begin{abstract}
This article presents a novel peak-to-average power ratio (PAPR) reduction scheme based on the insertion of dummy random Gaussian (IDRG) subcarriers to the data constellation points in the frequency domain integrated with a partial transmit sequence (PTS) technique. The dummy signals are Gaussian random signals which have the same statistical distribution as that of the output signals of the conventional orthogonal frequency division multiplexing (OFDM) system. The integration of IDRG with PTS technique reduces the overall complexity of the OFDM system. Compared to the conventional PTS which requires several inverse fast Fourier transform (IFFT) operations, the proposed IDRG-PTS scheme requires only half as many IFFT operations with comparable PAPR performance. Simulation results have been examined with 16 quadrate amplitude modulation OFDM signals.
\end{abstract}

Keyword: Orthogonal frequency division multiplexing; Insertion of dummy random Gaussian; Partial transmit sequence; Peak-to-average power ratio 